\title{
DILATONS IN FLAT AND CURVED SPACE-TIME
}

\author{
W. BUCHMÜLLER ${ }^{\mathrm{a}, \mathrm{b}}$ and N. DRAGON ${ }^{\mathrm{a} . \mathrm{c}}$ \\ ${ }^{a}$ Institut für Theoretische Phvsik, Universität Hannover, FRG \\ ${ }^{b}$ Deutsches Elektronen-Synchrotron DESY, Hamburg, FRG \\ 'Institut für Theoretische Physik, Universität Karlsruhe, FRG
}

Received 25 August 1988

\begin{abstract}
We investigate an extension of the standard model of strong and electroweak interactions with classical, nonlinearly realized, conformal invariance. The corresponding Goldstone boson, the dilaton, acquires a small mass due to the conformal anomaly. In curved space, the dilaton is identified with the conformal factor of the metric tensor which also contains the graviton. The corresponding action is invariant only under restricted coordinate transformations which preserve the volume. The theory has no cosmological term and the curvature of the ground state is determined by the vacuum expectation value of the dilaton field, which is an arbitrary integration constant of the gravitational field equations.
\end{abstract}

\section{Introduction}

Scale and conformal invariance play a central role in particle physics and quantum field theory ${ }^{\star}$. In string theories, which may lead to a unified description of all interactions, they appear as exact symmetries of the fundamental two-dimensional field theory [2]. In four-dimensional field theories conformal invariance is mostly of interest as an approximate symmetry, or as nonlinearly realized, "hidden" symmetry [3]. In both versions scale invariance has been extensively studied in connection with hadron physics $[3,4]$. In this paper, we will argue that "hidden" scale invariance may also be of interest as an approximate symmetry in connection with the standard model, the current theory of particle physics.

In the standard model of strong and electroweak interactions, the chiral nature of the fermion representation does not allow direct mass terms. Hence, the standard model lagrangian is classically invariant under dilatations, except for a single term, the mass parameter in the Higgs sector which, via the $S U(2) \times U(1)$ breaking vacuum expectation value, sets the scale of all particle masses. In many extensions of the standard model, such as supergravity models ${ }^{\star}$ the Higgs mass term itself is also a consequence of spontaneous symmetry breaking.

${ }^{\star}$ For a recent review and references, see ref. [1].

${ }^{\star}$ See, for instance, ref. [5]. 
Motivated by the approximate scale invariance of the standard model, we will investigate in the following a fully scale invariant extension which is obtained in the usual manner by introducing a Goldstone boson, the dilaton. This means that we consider the standard model as an effective low energy lagrangian of a more fundamental theory in which scale invariance is broken spontaneously. The appearance of dilatons is in fact a generic feature of all theories which are obtained from higher dimensional theories through compactification, in particular string theories [2].

Classical scale invariance implies that the Higgs potential of the theory with dilaton has a flat direction along which the vacuum expectation values are undetermined. The conformal anomaly lifts this vacuum degeneracy. It also generates a small mass for the dilaton, which becomes a pseudo-Goldstone boson, and determines the vacuum energy density.

A field theory with dilaton is almost identical to a theory where the dilaton is replaced by a conformally flat gravitational background field. This raises the question of the physical meaning of the dilaton in the presence of a gravitational background field, i.e., in curved space-time. We will discuss this problem in detail, in particular with respect to the conformal anomaly, and this will lead us to a version of Einstein's theory of gravity in which the invariance under diffeomorphisms is restricted to those coordinate transformations which preserve the volume, or alternatively, to a Brans-Dicke theory [6].

Many authors have speculated that in theories with dilatons, i.e., with spontaneously broken scale invariance, the cosmological constant might vanish. This would be analogous to the solution of the strong $C P$-problem of QCD in theories with axions, i.e., with spontaneously broken Peccei-Quinn symmetry [7]. Particularly interesting is the analogy between conformal anomaly and axial anomaly in both cases, and in recent work [8-10] the effect of the conformal anomaly on the cosmological constant has been studied. As we will see, the ground state curvature is indeed related to the vacuum expectation value of the dilaton field. However, spontaneously broken scale invariance does not lead to flat space-time as uniquely determined ground state.

The paper is organized as follows. In sect. 2, which is partly based on ref. [9], we discuss the standard model with dilaton, i.e., its minimal scale invariant extension. In particular, we derive the dilaton mass, which is generated by the conformal anomaly. Sect. 3 deals with dilatons in curved space-time. First we consider the classical theory with general coordinate invariance, and subsequently the effect of the conformal anomaly. Then we discuss the theory with restricted coordinate invariance, where the dilaton is identified with the conformal factor in the metric, and, as an alternative, the Brans-Dicke theory. Gauge fixing and ghost lagrangian are discussed in sect. 4 . Sect. 5 deals with the ground state curvature, and in sect. 6 we summarize our results. In the appendix, we discuss an ambiguity in the definition of the renormalized effective action in curved space-time. 


\section{The scale invariant standard model}

Quarks and leptons form a chiral representation of the electroweak gauge group $\mathrm{SU}(2) \times \mathrm{U}(1)$. As a consequence, direct fermion mass terms are not allowed, and the standard model lagrangian is almost entirely invariant under dilatations. This classical scale invariance is only broken by the mass parameters in the Higgs sector of the lagrangian which, including the electroweak gauge interactions of the Higgs doublet, reads:

$$
\begin{gathered}
L=L_{\text {gauge }}+L_{\mathrm{M}}, \\
L_{\text {gauge }}=-\frac{1}{4} W_{\mu \nu}^{\prime} W^{\prime \mu \nu}-\frac{1}{4} B_{\mu \nu} B^{\mu \nu}, \\
L_{\mathrm{M}}=-\mathrm{D}_{\mu} \varphi^{\dagger} \mathrm{D}^{\mu} \varphi-V_{0}\left(\varphi^{\dagger} \varphi\right), \\
V_{0}\left(\varphi^{\dagger} \varphi\right)=a^{4}+\mu^{2} \varphi^{\dagger} \varphi+(\lambda / 2)\left(\varphi^{\dagger} \varphi\right)^{2},
\end{gathered}
$$

where $a$ and $\mu$ are parameters of mass dimension 1 , and $\mathrm{D}_{\mu}, W_{\mu \nu}^{I}$ and $B_{\mu \nu}$ are the gauge covariant derivative and the $\mathrm{SU}(2)$ and $\mathrm{U}(1)$ field strengths respectively.

It is well known how to obtain a scale invariant extension [3] of the lagrangian (2.1). One introduces a dilaton field $\sigma(x)$ and multiplies the mass parameters in eq. (2.1d) by the appropriate exponential power of the dilaton field:

$$
a^{4} \rightarrow \bar{a}^{4}=a^{4} \mathrm{e}^{4 \sigma / f}, \quad \mu^{2} \rightarrow \bar{\mu}^{2}=\mu^{2} \mathrm{e}^{2 \sigma / f},
$$

where $f$ is the dilaton "decay constant". The derivative couplings of the dilaton field are not uniquely determined by the requirement of global scale invariance. We choose couplings to the Higgs doublet which, in a gravitational background field, yield maximal symmetry, i.e., invariance under local Weyl transformations:

$$
\delta \sigma(x)=f \Lambda(x), \quad \delta \varphi(x)=\Lambda(x) \varphi(x) .
$$

Together with the usual dilaton kinetic term [3] this yields for the scalar fields the lagrangian:

$$
\begin{aligned}
L_{\mathrm{M}}(\varphi, \sigma)= & -\frac{1}{2} \mathrm{e}^{2 \sigma / f} \partial_{\mu} \sigma \partial^{\mu} \sigma-\left(\mathrm{D}_{\mu}-\partial_{\mu}(\sigma / f)\right) \varphi^{\dagger}\left(\mathrm{D}^{\mu}-\partial^{\mu}(\sigma / f)\right) \varphi \\
& -a^{4} \mathrm{e}^{4 \sigma / f}-\mu^{2} \mathrm{e}^{2 \sigma / f} \varphi^{\dagger} \varphi-(\lambda / 2)\left(\varphi^{\dagger} \varphi\right)^{2} .
\end{aligned}
$$

The action (2.1), with (2.1c) replaced by (2.4), is invariant under dilatations,

$$
\begin{array}{rlrl}
\delta \sigma & =\delta \alpha\left(f+x^{\mu} \partial_{\mu} \sigma\right), & \delta \varphi & =\delta \alpha\left(\varphi+x^{\mu} \partial_{\mu} \varphi\right), \\
\delta W_{\mu}^{I} & =\delta \alpha\left(W_{\mu}^{I}+x^{\nu} \partial_{\nu} W_{\mu}^{I}\right), & \delta B_{\mu}=\delta \alpha\left(B_{\mu}+x^{\nu} \partial_{\nu} B_{\mu}\right),
\end{array}
$$


and the corresponding Noether current $S_{\mu}$ is conserved:

$$
\begin{gathered}
\delta \int \mathrm{d}^{4} x L=\delta \alpha \int \mathrm{d}^{4} x \partial^{\mu} S_{\mu}, \\
\partial^{\mu} S_{\mu}=0 .
\end{gathered}
$$

The couplings of the dilaton field are nonrenormalizable. Hence, the lagrangian (2.4) corresponds physically to a low energy effective lagrangian which is valid only for distances larger than $1 / f$, i.e., for energies below the mass scale at which scale invariance is spontaneously broken. In the following, we will therefore treat $\sigma(x)$ as a classical background field and compute quantum corrections only for the other matter and gauge fields which have renormalizable interactions. This is analogous to the treatment of quantum field theory in a gravitational background field.

It is convenient to use instead of the fields $\varphi$ and $\sigma$ the fields $\phi=\mathrm{e}^{-\sigma / f} \varphi$ and $\sigma$ in terms of which the lagrangian defined by eqs. (2.1) and (2.4) becomes

$$
\begin{aligned}
L=-\mathrm{e}^{4 \sigma / f}[ & \eta^{\mu \lambda} \eta^{\nu \tau} \mathrm{e}^{-4 \sigma / f}\left(\frac{1}{4} W_{\mu \nu}^{I} W_{\lambda \tau}^{I}+\frac{1}{4} B_{\mu \nu} B_{\lambda \tau}\right) \\
& \left.+\frac{1}{2} \eta^{\mu \nu} \mathrm{e}^{-2 \sigma / f} \partial_{\mu} \sigma \partial_{\nu} \sigma+\eta^{\mu \nu} \mathrm{e}^{-2 \sigma / f} \mathrm{D}_{\mu} \phi^{\dagger} \mathrm{D}_{\nu} \phi+V_{0}\left(\phi^{\dagger} \phi\right)\right]
\end{aligned}
$$

In this lagrangian the couplings of the dilaton field to other fields are identical to those of a conformally flat metric:

$$
\bar{g}_{\mu \nu}=\eta_{\mu \nu} \mathrm{e}^{2 \sigma / f}
$$

The only difference lies in the sign of the kinetic term: it is negative for the dilaton, corresponding to a physical degree of freedom, whereas the Einstein lagrangian for the gravitational field yields a positive sign for the kinetic term of the conformal factor.

From eq. (2.7), one immediately reads off the equation of motion for the dilaton field:

$$
\square \mathrm{e}^{\sigma / f}=\frac{1}{f^{2}} \mathrm{e}^{-\sigma / f} T_{\mu}^{\mu},
$$

where $T_{\mu \nu}$ is the energy-momentum tensor of the Higgs field:

$$
T_{\mu \nu}=-\mathrm{e}^{2 \sigma / f}\left(\mathrm{D}_{\mu} \phi^{\dagger} \mathrm{D}_{\nu} \phi+\mathrm{D}_{\nu} \phi^{\dagger} \mathrm{D}_{\mu} \phi\right)+\eta_{\mu \nu}\left(\mathrm{e}^{2 \sigma / f} \eta^{\lambda \tau} \mathrm{D}_{\lambda} \phi^{\dagger} D_{\tau} \phi+\mathrm{e}^{4 \sigma / f} V_{0}\left(\phi^{\dagger} \phi\right)\right)
$$


We note that eq. (2.9) is identical to the equation of motion for the scalar field in the Brans-Dicke theory [6].

In order to find the ground state of the theory, we have to solve the equations of motion for constant field configurations $\phi=\phi_{0}, \sigma=\sigma_{0}$. From eqs. (2.7) and (2.9) one easily obtains:

$$
\begin{aligned}
\left.\mathrm{e}^{4 \sigma / f} \frac{\partial}{\partial \phi^{\dagger}} V_{0}\left(\phi^{\dagger} \phi\right)\right|_{\phi=\phi_{0}, \sigma=\sigma_{0}} & =0, \\
\left.T^{\mu}{ }_{\mu}\right|_{\phi=\phi_{0}, \sigma=\sigma_{0}} & =4 \mathrm{e}^{4 \sigma_{0} / f} V_{0}\left(\phi_{0}^{\dagger} \phi_{0}\right)=0 .
\end{aligned}
$$

In the case $\mu^{2}<0$ eq. (2.11a) has the usual, $\mathrm{SU}(2) \times U(1)$ breaking solution $\phi_{0} \neq 0$. Eq. (2.11b) has a solution with finite $\sigma_{0}$ only, if the potential vanishes at the minimum. This determines the constant $a^{4}$ in $V_{0}$ as $\mu^{4} / 2 \lambda$, i.e., one has

$$
V\left(\phi^{\dagger} \phi\right)=(\lambda / 2)\left(\phi^{\dagger} \phi+\mu^{2} / \lambda\right)^{2}
$$

for the classical Higgs potential. Then the vacuum expectation value $\sigma_{0}$ of the dilaton field is undetermined, corresponding to the "flat direction" of the potential which is always associated with classical scale invariance [11].

In ref. [9], it has been shown that this vacuum degeneracy is lifted by quantum corrections. The complete one-loop effective potential reads [9]:

$$
V\left(\phi^{\dagger} \phi, \sigma\right)=\mathrm{e}^{4 \sigma / f}\left(\bar{V}\left(\phi^{\dagger} \phi\right)-\Delta\left(\phi^{\dagger} \phi\right) \sigma / f\right),
$$

where $\vec{V}\left(\phi^{\dagger} \phi\right)$ is the complete Coleman-Weinberg potential [12] with coupling constants defined in a minimal subtraction scheme $\left(z=\phi^{\dagger} \phi\right)$ :

$$
\begin{array}{r}
\vec{V}(z)=a^{4}+\mu^{2} z+(\lambda / 2) z^{2}+\frac{1}{(8 \pi)^{2}}\left[\frac{3}{2}\left(\mu^{2}+\lambda z\right)^{2}\left(\ln \frac{\left(\mu^{2}+\lambda z\right)^{2}}{M^{4}}-1\right)\right. \\
+\frac{1}{2}\left(\mu^{2}+3 \lambda z\right)^{2}\left(\ln \frac{\left(\mu^{2}+3 \lambda z\right)^{2}}{M^{4}}-1\right)+\frac{3}{2} g^{4} z^{2}\left(\ln \frac{g^{2} z}{2 M^{2}}-\frac{1}{2}\right) \\
\left.+\frac{3}{4}\left(g^{2}+g^{\prime 2}\right)^{2} z^{2}\left(\ln \frac{\left(g^{2}+g^{\prime 2}\right) z}{2 M^{2}}-\frac{1}{2}\right)\right] \cdot(2.14)
\end{array}
$$

$\Delta(z)$ is the contribution to the conformal anomaly [13] without derivatives, which 
can be read off from $\bar{V}(z)$ :

$$
\Delta(z)=M \partial \bar{V}(z) / \partial M
$$

where $M$ is the renormalization mass on which $V$ depends logarithmically.

The conditions for an extremum of the potential (2.13) read:

$$
\begin{aligned}
& \mathrm{e}^{4 \sigma_{0} / f} \frac{\partial}{\partial \phi^{\dagger}}\left(\bar{V}\left(\phi^{\dagger} \phi\right)-\Delta\left(\phi^{\dagger} \phi\right) \frac{\sigma_{0}}{f}\right)_{\phi=\phi_{0}}=0 \\
& \mathrm{e}^{4 \sigma_{0} / f}\left(\bar{V}\left(\phi_{0}^{\dagger} \phi_{0}\right)-\Delta\left(\phi_{0}^{\dagger} \phi_{0}\right)\left(\frac{\sigma_{0}}{f}+\frac{1}{4}\right)\right)=0 .
\end{aligned}
$$

If the parameters of the potential, which depend now on the renormalization mass, satisfy the condition

$$
\frac{2 \lambda(M) a^{4}(M)-\mu^{4}(M)}{\mu^{4}(M)}=\mathrm{O}\left(\lambda, g^{4}, g^{\prime 4}\right)
$$

a solution of (2.16) exists [9] with finite $\sigma_{0}$ which, by means of a redefinition of the parameters $a$ and $\mu$, can always be arranged to be at $\sigma_{0}=0$. Then one easily obtains from (2.13) the dilaton mass to leading order in $1 / f$ [9]:

$$
\begin{aligned}
m_{\mathrm{D}}^{2} & =-\left(4 / f^{2}\right) \Delta\left(\phi_{0}^{\dagger} \phi_{0}\right) \\
& =\left(1 / 8 \pi^{2} f^{2}\right)\left(6 m_{\mathrm{W}}^{4}+3 m_{\mathrm{Z}}^{4}+m_{\mathrm{H}}^{4}\right) .
\end{aligned}
$$

The general expression, which includes contributions from fermion loops, is given by the supertrace of the mass operator $\mathscr{M}$ (for the effect of mass scales beyond the standard model, see ref. [23]):

$$
\begin{aligned}
m_{\mathrm{D}}^{2} & =\left(1 / 8 \pi^{2} f^{2}\right) \operatorname{Str} \mathscr{M}^{4} \\
& =1 / 8 \pi^{2} f^{2}\left(\sum_{\text {bosons }} m_{\mathrm{b}}^{4}-\sum_{\text {fermions }} m_{\mathrm{f}}^{4}\right) .
\end{aligned}
$$

In the standard model the t-quark contributes $-4 m_{\mathrm{t}}^{4}$ inside the bracket of eq. (2.18). For $\sigma_{0}=0$, the Higgs mass is given by

$$
m_{\mathrm{H}}^{2}=\left.4 z \frac{\partial^{2} \bar{V}}{\partial z^{2}}\right|_{z=\phi_{0}^{\dagger} \phi_{0}},
$$


which satisfies the Weinberg-Linde bound [14]

$$
m_{\mathbf{H}}^{2} \geqslant \frac{\sqrt{2} G_{\mathrm{F}}}{l 6 \pi^{2}}\left(6 m_{\mathrm{W}}^{4}+3 m_{\mathrm{Z}}^{4}\right),
$$

where $G_{\mathrm{F}}$ is Fermi's constant. From eq. (2.19), we conclude that the one-loop stability of the vacuum requires fermion masses to be smaller than the largest boson mass in the theory.

From eq. (2.13) one also obtains an important relation between the vacuum energy density and the conformal anomaly. The equation of motion for $\sigma$ yields with $\sigma_{0}=0$ :

$$
\begin{aligned}
V\left(\phi_{0}^{\dagger} \phi_{0}, \sigma_{0}\right) & =\frac{1}{4} \Delta\left(\phi_{0}^{\dagger} \phi_{0}\right) \\
& =-\frac{1}{16} f^{2} m_{\mathrm{D}}^{2}<0 .
\end{aligned}
$$

This means that, contrary to ordinary field theories in flat space, in theories with a dilaton field the vacuum energy density is not an arbitrary parameter. In particular, if the gravitational field is coupled to matter in the usual manner, flat space-time cannot be a solution of Einstein's equations since the ground state curvature is proportional to the vacuum energy density, which does not vanish. However, the relation between curvature scalar and trace of the energy-momentum tensor follows from the variation of the action with respect to the conformal factor of the metric. Since for constant fields the couplings of dilaton field and conformal factor are identical and an extremum of the action exists with $\sigma=\sigma_{0}=$ const. in flat space, one might expect that in theories with dilatons the usual relation between ground state curvature and vacuum energy density should be modified. We will pursue this question in the following sections.

Quantum corrections break the conformal invariance of the classical action. From eq. (2.13) one easily derives that the divergence of the dilatation current is given by the conformal anomaly:

$$
\partial^{\mu} S_{\mu}=\Delta .
$$

If a theory contains the Goldstone boson of an anomalous symmetry, the symmetry can be restored by means of a local term, the corresponding Wess-Zumino term [15]. In our case, this term is

$$
\Gamma_{\mathrm{WZ}}=-\int \mathrm{d}^{4} x \mathrm{e}^{4 \sigma / f} \Delta\left(\phi^{\dagger} \phi\right)(\sigma / f),
$$

i.e., one simply subtracts the anomaly term in eq. (2.13). The resulting effective action is scale invariant, but physically unacceptable unless the constant term of the potential (2.1d) is fine-tuned such that $\bar{V}\left(\phi^{\dagger} \phi\right)$ vanishes at the minimum, i.e., 
$\bar{V}\left(\phi_{0}^{\dagger} \phi_{0}\right)=0$. Otherwise, the only constant solution of the equations of motion is obtained for $\sigma_{0} \rightarrow-\infty$. We conclude that in theories with dilatons the conformal anomaly should be kept. This is required for the existence of the ground state, unless one accepts fine-tuning of the constant term in the effective potential.

\section{Dilatons in curved space-time}

The extension of the model considered in the previous sections to curved space appears to be straightforward. The gravitational background field is incorporated in the standard manner by replacing in the lagrangian (2.7) the flat-space metric $\eta_{\mu \nu}$ by an arbitrary metric tensor $g_{\mu \nu}$ :

$$
\begin{aligned}
\sqrt{g} L= & -\sqrt{g}\left(\frac{1}{2} g^{\mu \nu} \mathrm{e}^{(2 \sigma / f)} \partial_{\mu} \sigma \partial_{\nu} \sigma-L_{\mathrm{M}}\right), \\
L_{m}= & -\frac{1}{4} g^{\mu \lambda} g^{\nu \tau}\left(W_{\mu \nu}^{I} W_{\lambda \tau}^{I}+B_{\mu \nu} B_{\lambda \tau}\right) \\
& -g^{\mu \nu} \mathrm{e}^{2 \sigma^{\prime} f} \mathrm{D}_{\mu} \phi^{\dagger} \mathrm{D}_{\nu} \phi-\mathrm{e}^{4 \sigma^{\prime} f} \bar{V}_{0}\left(\phi^{\dagger} \phi\right) .
\end{aligned}
$$

The corresponding action is invariant under general coordinate transformations and global Weyl transformations:

$$
\delta \boldsymbol{\sigma}(x)=f \Lambda, \quad \delta g_{\mu \nu}(x)=2 \Lambda g_{\mu \nu}(x) .
$$

Demanding Weyl invariance also for the kinetic term of the gravitational field one obtains for the complete lagrangian

$$
\begin{aligned}
\sqrt{g} L & =\sqrt{g}\left(L_{\mathrm{G}}+L_{\mathrm{M}}\right), \\
L_{\mathrm{G}} & =-\frac{1}{2} \kappa \mathrm{e}^{2 \sigma / f} R-\frac{1}{2} g^{\mu \nu} \mathrm{e}^{2 \sigma / f} \partial_{\mu} \sigma \partial_{\nu} \sigma,
\end{aligned}
$$

where $L_{\mathrm{M}}$ is the lagrangian (3.1b) for Higgs and gauge fields, $R$ is the curvature scalar, and $1 / 8 \pi \kappa \equiv G_{\mathrm{N}}$ is Newton's constant. $\kappa$ has mass dimension 2 and is therefore multiplied by $\mathrm{e}^{2 \sigma / f}$.

The gravitational part of the lagrangian, which is given by (3.3b), is identical to that of the Brans-Dicke theory [6], with the identifications $\chi=(\kappa / 2) \mathrm{e}^{2 \sigma / f}, \omega=$ $f^{2} / 4 \kappa$, where $\chi$ is the Brans-Dicke scalar field. However, contrary to the Brans-Dicke theory, in the lagrangian (3.3) the field $\sigma(x)$ couples also to other scalar and gauge fields. As these interactions depend only on the product

$$
\bar{g}_{\mu \nu}=g_{\mu \nu} \mathrm{e}^{2 \sigma / f},
$$


the lagrangian (3.3) can be rewritten in the form (cf. (3.19))

$$
\begin{aligned}
-\sqrt{g} L & =\sqrt{\bar{g}}\left(\kappa / 2 \bar{R}+\frac{1}{2}\left(1+6 \kappa / f^{2}\right) \bar{g}^{\mu \nu} \partial_{\mu} \sigma \partial_{\nu} \sigma+\bar{g}^{\mu \nu} \mathrm{D}_{\mu} \phi^{\dagger} \mathrm{D}_{\nu} \phi+V_{0}\left(\phi^{\dagger} \phi\right)\right) \\
& \equiv-\sqrt{\bar{g}} \bar{L}
\end{aligned}
$$

Here the "dilaton" has become a free field because in eq. (2.4), the interactions between $\sigma$ and $\varphi$ have been chosen to yield local Weyl invariance. In the more general case of global Weyl invariance only, additional derivative couplings between $\phi$ and $\sigma$ occur which, however, are not important for the following discussion.

The action corresponding to the lagrangian (3.5) is invariant under general coordinate transformations with respect to the metric $\bar{g}_{\mu \nu}$, and the dilaton appears as Goldstone boson of the Weyl transformation (3.2):

$$
\sigma(x) \rightarrow \sigma(x)+f \Lambda
$$

This corresponds to the fact that dilatations can be viewed as special coordinate transformations with an accompanying global Weyl transformations which acts trivially on the new metric $\bar{g}_{\mu \nu}$.

Quantum corrections will in general break the invariance of the lagrangian (3.5) under general coordinate transformations and global Weyl transformations because of the conformal anomaly. However, the quantum effective action is only defined up to local terms which may be nonrenormalizable with respect to the interactions of dilaton and gravitational fields. In the case of local Weyl invariance this ambiguity has been discussed by Bonora, Pasti and Tonin [16]. Requiring general coordinate invariance one is led to the effective potential (cf. ref. [8]):

$$
\sqrt{g} V\left(\phi^{\dagger} \phi, \sigma\right)=\sqrt{g} \mathrm{e}^{4 \sigma / f}\left(\vec{V}\left(\phi^{\dagger} \phi\right)-\Delta\left(\phi^{\dagger} \phi\right) \sigma / f\right) .
$$

On the other hand, since the couplings of $g_{\mu \nu}$ and $\sigma$ to other fields depend only on the product $\bar{g}_{\mu \nu}=\mathrm{e}^{2 \sigma / f} g_{\mu \nu}$, a straightforward calculation yields [9]:

$$
\begin{aligned}
\sqrt{g} V\left(\phi^{\dagger} \phi, \sigma, \sqrt{g}\right) & =\sqrt{g} \mathrm{e}^{4 \sigma / f}\left(\bar{V}\left(\phi^{\dagger} \phi\right)-\Delta\left(\phi^{\dagger} \phi\right)\left(\sigma / f+\frac{1}{4} \ln \sqrt{g}\right)\right) \\
& =\sqrt{\bar{g}}\left(\bar{V}\left(\phi^{\dagger} \phi\right)-\frac{1}{4} \Delta\left(\phi^{\dagger} \phi\right) \ln \sqrt{\bar{g}}\right) .
\end{aligned}
$$

In eq. (3.7) general coordinate invariance is maintained and Weyl invariance is broken whereas in eq. (3.8) Weyl invariance is kept and general coordinate invariance is broken. 
In the latter case, the local symmetry is restricted to coordinate transformations, under which the volume element $\sqrt{g}$ transforms as a scalar field. This means that the gauge parameter $\varepsilon_{\mu}$ which appears in the Lie derivatives

$$
\begin{aligned}
\delta \phi & =\varepsilon^{\mu} \partial_{\mu} \phi, \\
\delta g_{\mu \nu} & =\varepsilon^{\lambda} \partial_{\lambda} g_{\mu \nu}+\partial_{\mu} \varepsilon^{\lambda} g_{\lambda \nu}+\partial_{\nu} \varepsilon^{\lambda} g_{\mu \lambda} \\
\delta \sqrt{g} & =\partial_{\mu}\left(\sqrt{g} \varepsilon^{\mu}\right)
\end{aligned}
$$

has to be transverse:

$$
\partial_{\mu} \varepsilon^{\mu}=0
$$

One then has

$$
\delta \sqrt{g}=\varepsilon^{\mu} \partial_{\mu} \sqrt{g} .
$$

The transversality condition (3.10) selects volume preserving diffeomorphisms.

Both definitions for the renormalized effective potential, eq. (3.7) and eq. (3.8), are physically unacceptable if the kinetic term is given by eq. (3.3b). In the first case one easily derives from the equations of motion

$$
\begin{aligned}
\delta \zeta / \delta \sigma & =0, \\
g^{\mu \nu} \delta \zeta / \delta g^{\mu \nu} & =0,
\end{aligned}
$$

that the ground state $\left(\phi_{0}, \sigma_{0}\right)$ has to satisfy

$$
\mathrm{e}^{4 \sigma_{0} / f} \Delta\left(\phi_{0}^{\dagger} \phi_{0}\right)=0 .
$$

At least in the standard model, where the vacuum expectation value of the anomaly does not vanish, this would lead to $\sigma_{0} \rightarrow-\infty$. In the second case, given by eq. (3.8), one concludes from eq. (3.5) that, in addition to the free field $\sigma$, the theory contains a massive ghost corresponding to $\ln \sqrt{\bar{g}}$ which, since general coordinate invariance is broken, is an unacceptable physical degree of freedom. This problem can be evaded in a particular class of gauges [17] where the anomaly term $\Delta \ln \sqrt{g}$ can be interpreted as radiative correction to the gauge fixing lagrangian. However, in this "volume gauge" the separation between physical dilaton and the conformal factor in the metric is not very transparent.

All these problems are avoided if dilaton and graviton are described together by the symmetric tensor field $g_{\mu \nu}{ }^{\star}$ and a lagrangian whose local symmetry is restricted to volume preserving diffeomorphisms. The kinetic terms for graviton and dilaton

\footnotetext{
* This corresponds to the construction of graviton and dilaton states in string theories [18]
} 
are given by

$$
-\sqrt{g} L_{\mathrm{G}}=\sqrt{g}\left(\frac{\kappa}{2} R+\frac{f^{2}}{2} g^{\mu \nu} \frac{1}{\sqrt{g}^{1 / 2}} \partial_{\mu} \sqrt{g}^{-1 / 4} \partial_{\nu} \sqrt{g}^{1 / 4}\right) .
$$

Here the field $\sigma$ has been identified with $(f / 4) \ln \sqrt{g}$ which transforms inhomogeneously under dilatations $\delta x^{\mu}=\varepsilon^{\mu}=\lambda x^{\mu}$ :

$$
\delta((f / 4) \ln \sqrt{g})=\lambda\left(f+x^{\mu} \partial_{\mu}(f / 4) \ln \sqrt{g}\right) .
$$

The lagrangian (3.14) is uniquely defined by demanding invariance under the transformations (3.9) with

$$
\partial^{\mu} \varepsilon_{\mu}=c
$$

where $c$ is an arbitrary constant. The condition (3.16) defines a subgroup of the group of general coordinate transformations which contains volume preserving transformations satisfying eq. (3.10) and global dilatations. This invariance determines the couplings of $g_{\mu \nu}$ to other fields to be the same as in the case of general coordinate invariance, i.e., one obtains for the matter and gauge part of the lagrangian (3.1b). Since there is only one "dilaton field", namely $\ln \sqrt{g}$, the conformal anomaly can only be incorporated in the form (3.8) and we obtain as complete lagrangian

$$
\begin{gathered}
\sqrt{g} L=\sqrt{g}\left(L_{\mathrm{G}}+L_{\mathrm{M}}+L_{\mathrm{AN}}\right) \\
L_{\mathrm{G}}=-\frac{\kappa}{2} R-\frac{f^{2}}{2} g^{\mu \nu} \frac{1}{\sqrt{g}} \partial_{\mu} \sqrt{g}^{1 / 4} \partial_{\nu} \sqrt{g}^{1 / 4}, \\
L_{\mathrm{M}}=-\frac{1}{4} g^{\mu \lambda} g^{\mu \tau}\left(W_{\mu \nu}^{I} W_{\lambda \tau}^{I}+B_{\mu \nu} B_{\lambda \tau}\right)-g^{\mu \nu} \mathrm{D}_{\mu} \phi^{\dagger} \mathrm{D}_{\nu} \phi-\bar{V}\left(\phi^{\dagger} \phi\right), \\
L_{\mathrm{AN}}=\frac{1}{4} \Delta\left(\phi^{\dagger} \phi\right) \ln \sqrt{g}
\end{gathered}
$$

where $\bar{V}$ is given by eq. (2.14). We emphasize that the particular form of the potential for $\sqrt{g}$ has its origin in the identification of $(f / 4) \ln \sqrt{g}$ with a physical dilaton field.

The lagrangian (3.17) is only invariant under restricted coordinate transformations defined by eqs. (3.9) and (3.10). With respect to these transformations $\sqrt{g}$ is a scalar field (cf. (3.11)), and it is instructive to express the lagrangian (3.17) in terms of $\sigma=(f / 2) \ln \sqrt{g}$ and a metric $\hat{g}_{\mu \nu}$ with determinant $(-1)$ :

$$
g_{\mu \nu}=\mathrm{e}^{2 \sigma / f} \hat{g}_{\mu \nu}, \quad \operatorname{det}\left(\hat{g}_{\mu \nu}\right)=-1
$$


Using the identity

$$
R=\mathrm{e}^{-2 \sigma / f}\left[\hat{R}+6 \frac{1}{\sqrt{\hat{g}}} \partial_{\mu}\left(\hat{g}^{\mu \nu} \sqrt{\hat{g}} \partial_{\nu} \sigma\right)+6 \hat{g}^{\mu \nu} \partial_{\mu} \sigma \partial_{\nu} \sigma\right]
$$

one easily finds

$$
\begin{aligned}
-\sqrt{g} L=\sqrt{\hat{g}}[ & \frac{\kappa}{2} \mathrm{e}^{2 \sigma / f} \hat{R}+\frac{1}{2}\left(1-6 \frac{\kappa}{f^{2}}\right) \hat{g}^{\mu \nu} \mathrm{e}^{2 \sigma / f} \partial_{\mu} \sigma \partial_{\nu} \sigma \\
& +\frac{1}{4} \hat{g}^{\mu \lambda} \hat{g}^{\nu \tau}\left(W_{\mu \nu}^{I} W_{\lambda \tau}^{I}+B_{\mu \nu} B_{\lambda \tau}\right)+\hat{g}^{\mu \nu} \mathrm{e}^{2 \sigma / f} \mathrm{D}_{\mu} \phi^{\dagger} \mathrm{D}_{\nu} \phi \\
& \left.+\mathrm{e}^{4 \sigma / f}\left(\bar{V}\left(\phi^{\dagger} \phi\right)-\Delta\left(\phi^{\dagger} \phi\right) \sigma / f\right)\right]+\Lambda(\sqrt{\hat{g}}-1) .
\end{aligned}
$$

Here $\Lambda(x)$ is a Lagrange multiplier field which enforces the constraint (3.18) for the metric tensor $\hat{g}_{\mu \nu}$.

The theory of gravity contained in the lagrangian $(3.20)$ is known $[19,20]$ to be classically equivalent to Einstein's theory of gravity which is invariant under general coordinate transformations. The only difference concerns the role of the cosmological constant. Clearly, in the theory defined by (3.20) a cosmological term has no meaning, it simply redefines the Lagrange multiplier field $\Lambda(x)$. However, as discussed in refs. $[19,20]$, the cosmological term reappears as an integration constant of the equations of motion which determines the ground state curvature. We will see in sect. 5 that in our particular model with dilaton, this constant is related to the vacuum expectation value $\sigma_{0}$ of the dilaton field $\sigma(x)$.

The lagrangian (3.20) can be viewed as a general covariant theory in a special class of gauges for the gravitational field, which is defined through the constraint $\sqrt{g}=1$. In the following section, we will discuss the complete gauge fixing and ghost lagrangian for this theory.

So far we have discussed the possibility to extend the concept of the dilaton as a physical degree of freedom, which transforms inhomogeneously under dilatations, to curved space-time. This turned out to be possible, at least within a theory whose general coordinate invariance is restricted to volume preserving transformations. However, this whole ansatz may not be relevant for effective low energy lagrangians of more fundamental theories, such as string theories. All essential features of the flat space model described in sect. 2 are also reproduced by a theory with general coordinate invariance where Weyl invariance is restricted to the matter part of the lagrangian. In this case, the lagrangian (3.1b) for Higgs and gauge fields would be supplemented by the non-Weyl-invariant kinetic terms

$$
\sqrt{g} L_{\mathrm{G}}=-\sqrt{g}\left(\frac{1}{2} \kappa R+\frac{1}{2} g^{\mu v} \partial_{\mu} \sigma \partial_{\nu} \sigma\right) .
$$


In this case, the anomaly term in eq. (3.7) does not lead to the unwanted condition (3.13).

The lagrangian given by eqs. (3.1b) and (3.21) defines precisely a Brans-Dicke theory [6] which appears to be the correct effective field theory for graviton and "dilaton" arising in string theories [18]. We will come back to this model in sect. 5.

\section{Gauge fixing for restricted coordinate invariance}

In ref. [17], we have shown that the condition

$$
C(\sqrt{\hat{g}})=\sqrt{\hat{g}}-1=0
$$

can be part of a complete gauge fixing of a general covariant theory. The necessary three additional conditions may be chosen as (cf. ref. [21])

$$
C_{\mu \nu}(\hat{g})=\partial_{\mu} C_{\nu}(\hat{g})-\partial_{\nu} C_{\mu}(\hat{g})=0
$$

with

$$
C_{\mu}(\hat{g})=-\frac{1}{\sqrt{\hat{g}}} \hat{g}_{\mu \nu} \partial_{\lambda}\left(\sqrt{\hat{g}} \hat{g}^{\nu \lambda}\right)
$$

The corresponding gauge fixing lagrangian reads

$$
L_{\mathrm{GF}}=-(\alpha / 4) C^{\mu \nu} C_{\mu \nu}-(\beta / 2) C^{2},
$$

where indices are raised with the flat metric $\eta_{\mu \nu}$. In the limit $\beta \rightarrow \infty$ the second term in (4.4) becomes a constraint,

$$
-\frac{1}{2} \beta C^{2} \rightarrow-\Lambda C
$$

where $\Lambda$ is the corresponding Lagrange multiplier field which also occurs in eq. (3.20)

The ghost system ${ }^{\star}$ can also easily be obtained from ref. [17]. It contains 5 ghosts and 5 anti-ghosts, $u^{\mu}, v, \bar{u}^{\mu}$, and $\bar{v}$ where $v$ and $\bar{v}$ enforce the transversality of the vector ghosts $u^{\mu}$ and $\bar{u}^{\mu}$. The nilpotent BRS operator $s$ acts on scalar and vector fields as the Lie derivative where the gauge parameter $\varepsilon^{\mu}$ is replaced by the ghost $u^{\mu}$. For the metric tensor, the Lagrange multiplier field and the ghosts, the BRS

* An alternative ghost system has been constructed in ref. [21]. 
transformation reads:

$$
\begin{aligned}
s \hat{g}_{\mu \nu} & =u^{\lambda} \partial_{\lambda} \hat{g}_{\mu \nu}+\partial_{\mu} u^{\lambda} \hat{g}_{\lambda \nu}+\partial_{\nu} u^{\lambda} \hat{g}_{\mu \lambda}, \\
s \Lambda & =u^{\mu} \partial_{\mu} \Lambda, \\
s u^{\mu} & =u^{\nu} \partial_{\nu} u^{\mu}, \\
s v & =0, \\
s \bar{u}^{\mu} & =i \alpha \partial_{\nu} C^{\nu \mu}, \\
s \bar{v} & =-i \Lambda .
\end{aligned}
$$

The ghost lagrangian is given by

$$
L_{\mathrm{GH}}=i \bar{u}^{\mu} s C_{\mu}+i \bar{u}^{\mu} \partial_{\mu} v+i \bar{w} s C .
$$

It is identical to the ghost lagrangian for the "volume gauge" with finite $\beta$ (cf. eq. (4.4)). One easily verifies the invariance under BRS transformations for the sum of ghost and gauge fixing lagrangians:

$$
s \int \mathrm{d}^{4} x\left(L_{\mathrm{GF}}+L_{\mathrm{GH}}\right)=0,
$$

with

$$
L_{\mathrm{GF}}=-\frac{1}{4} \alpha C^{\mu \nu} C_{\mu \nu}-\Lambda C .
$$

We note that on the anti-ghosts $\bar{u}^{\mu}$ and $\bar{v}$ the BRS operator is nilpotent only "on-shell", i.e., for

$$
\begin{array}{r}
s \partial_{\nu} C^{\nu \mu}=0, \\
s \Lambda=u^{\mu} \partial_{\mu} \Lambda=0 .
\end{array}
$$

The first equation follows from the ghost equation of motion, the second equation is equivalent to the covariant conservation of the energy-momentum tensor [20] and follows from the equation of motion for the metric tensor in the case of restricted coordinate invariance.

We have seen that gauge fixing and ghost lagrangians of a theory with restricted coordinate invariance are identical with those of a theory with general coordinate invariance in a particular class of gauges where the volume element is fixed through the constraint $\sqrt{\hat{g}}=1$. So far, we have employed the field variables $\hat{g}_{\mu \nu}, \sigma$ and the Lagrange multiplier field $\Lambda$. However, knowing the ghost lagrangian and the BRS 
algebra, a description in terms of the variables $g_{\mu \nu}=\mathrm{e}^{2 \sigma / f} \hat{g}_{\mu \nu}, \sigma$ and $\Lambda$ is more economical, because the fields $\sigma$ and $\Lambda$ can then be eliminated by means of their equations of motion. The part of the lagrangian which depends on $\sigma$ and $A$ is given by (cf. eq. (3.20)):

$$
L_{\sigma, \Lambda}=-\frac{1}{2} g^{\mu \nu} \partial_{\mu} \sigma \partial_{\nu} \sigma+\Delta\left(\phi^{\dagger} \phi\right) \sigma / f-\Lambda\left(e^{-4 \sigma / f}-\sqrt{g}^{-1}\right) .
$$

The equations of motion for $\sigma$ and $A$ read:

$$
\begin{gathered}
\mathrm{e}^{4 \sigma / f}=\sqrt{g}, \\
\square \sigma+(1 / f) \Delta+(4 / f) \Lambda \mathrm{e}^{-4 \sigma / f}=0,
\end{gathered}
$$

where

$$
\square=\frac{1}{\sqrt{g}} \partial_{\mu}\left(\sqrt{g} g^{\mu \nu} \partial_{\nu}\right)
$$

They determine both fields in terms of the metric tensor:

$$
\begin{aligned}
& \boldsymbol{\sigma}=(f / 4) \ln \sqrt{g}, \\
& \Lambda=-\frac{1}{4} \sqrt{g}\left(\Delta+f^{2} \square \ln \sqrt{g}^{1 / 4}\right) .
\end{aligned}
$$

Hence one obtains

$$
L_{\sigma . \Lambda}=-\frac{f^{2}}{2} g^{\mu \nu} \frac{1}{\sqrt{g}^{1 / 2}} \partial_{\mu} \sqrt{g}^{1 / 4} \partial_{\nu} \sqrt{g}^{1 / 4}+\frac{1}{4} \Delta\left(\phi^{\dagger} \phi\right) \ln \sqrt{g}
$$

which, together with curvature scalar and matter part $L_{\mathrm{M}}$, yields the complete lagrangian (3.17). Eq. (4.16) is important for the BRS algebra. After eliminating the field $\Lambda$ eqs. (4.6b) and (4.6f) become

$$
\begin{aligned}
& s \bar{v}=+(i / 4) \sqrt{g}\left(\Delta+f^{2} \square \ln \sqrt{g}^{1 / 4}\right) . \\
& s^{2} \bar{v}=+(i / 4) u^{\mu} \partial_{\mu}\left(\sqrt{g}\left(\Delta+f^{2} \square \ln \sqrt{g}^{1 / 4}\right)\right) .
\end{aligned}
$$

In order to obtain the last equation from (4.6a) one has to use the transversality of $u^{\mu}$ which follows from the equation of motion for $\bar{v}$. In sect. 5 (cf. eq. (5.5)), we shall see that the necessary condition $s^{2} \bar{v}=0$ is indeed satisfied for solutions of the gravitational field equations.

For the complete graviton-dilaton lagrangian including the gauge fixing term, we can now identify physical states and masses as poles of the propagator matrix. From 
the kinetic term (cf. (3.17), (4.4))

$$
L_{\mathrm{kin}}=-\sqrt{g}\left(\frac{\kappa}{2} R+\frac{1}{2} f^{2} g^{\mu \nu} \frac{1}{\sqrt{g}^{1 / 2}} \partial_{\mu} \sqrt{g}^{1 / 4} \partial_{\nu} \sqrt{g}^{1 / 4}\right)-\frac{\alpha}{4} C^{\mu \nu} C_{\mu \nu},
$$

one obtains in an expansion around flat space,

$$
g_{\mu \nu}=\eta_{\mu \nu}+\kappa^{-1 / 2} h_{\mu \nu},
$$

for the quadratic part in $h_{\mu \nu}$

$$
\begin{aligned}
L_{\text {kin }}^{(2)}= & -\frac{1}{8}\left(\partial_{\mu} h_{\nu \rho}\right)^{2}+\frac{1}{4}\left(\partial^{\lambda} h_{\lambda \mu}\right)^{2}+\frac{1}{4} h_{\lambda}^{\lambda} \partial^{\tau} \partial^{\sigma} h_{\tau \sigma}-\frac{\gamma-1}{8}\left(\partial_{\mu} h_{\lambda}^{\lambda}\right)^{2} \\
& -\frac{\alpha}{4 \kappa}\left(\partial_{\mu} \partial^{\lambda} h_{\lambda \nu}-\partial_{\nu} \partial^{\lambda} h_{\lambda \mu}\right)^{2}+\frac{1}{32} \frac{\Delta_{0}}{\kappa}\left(h_{\lambda}^{\lambda}\right)^{2}, \quad \gamma=\frac{f^{2}}{16 \kappa} .
\end{aligned}
$$

Here we have added a "mass term" for $h^{\lambda}$ which, as we have seen in sect. 2, arises after spontaneous symmetry breaking. $\Delta_{0} \equiv \Delta\left(\phi_{0}^{\dagger} \phi_{0}\right)$ is the vacuum expectation value of the anomaly.

From the lagrangian (4.22) one obtains, after a straightforward calculation, the propagator:

$$
\left\langle h_{\mu \nu}(x) h_{\lambda \tau}(y)\right\rangle_{0}=4\left(P_{\mu \nu \lambda \tau}^{(2)} \frac{1}{\square}+\frac{1}{\gamma} P_{\mu \nu \lambda \tau}^{(0)} \frac{1}{\square-m^{2}}\right) \delta^{4}(x-y),
$$

where

$$
\begin{aligned}
P_{\mu \nu \lambda \tau}^{(2)}= & \frac{1}{2}\left(\eta_{\mu \lambda} \eta_{\nu \tau}+\eta_{\mu \tau} \eta_{\nu \lambda}-\eta_{\mu \nu} \eta_{\lambda \tau}\right)-\frac{1}{2}\left(1+\frac{\kappa}{2 \alpha} \frac{1}{\square}\right) \\
& \times\left(\eta_{\mu \lambda} \partial_{\nu} \partial_{\tau}+\eta_{\nu \lambda} \partial_{\mu} \partial_{\tau}+\eta_{\mu \tau} \partial_{\nu} \partial_{\lambda}+\eta_{\nu \tau} \partial_{\mu} \partial_{\lambda}-4 \partial_{\mu} \partial_{\nu} \partial_{\lambda} \partial_{\tau} / \square\right) / \square \\
& +\left(\eta_{\mu \nu} \partial_{\lambda} \partial_{\tau}+\partial_{\mu} \partial_{\nu} \eta_{\lambda \tau}\right) / \square-4 \partial_{\mu} \partial_{\nu} \partial_{\lambda} \partial_{\tau} / \square^{2} \\
P_{\mu \nu \lambda \tau}^{(0)}= & \partial_{\mu} \partial_{\nu} \partial_{\lambda} \partial_{\tau} / \square^{2} \\
m^{2}= & -\Delta_{0} / 8 \gamma \kappa=-4 \Delta_{0} / f^{2}
\end{aligned}
$$

The propagator (4.23) agrees with the one obtained in ref. [21]. It has two poles, at $k^{2}=0$ and at $k^{2}=-m^{2}$, where $m$ is precisely the dilaton mass (cf. eq. (2.18)) which we have calculated in flat space in sect. 2 . 
In order to verify that the two poles of the propagator correspond to two different states of spin 0 and 2 , we consider the plane waves

$$
\begin{array}{rlrl}
D_{\mu \nu} & =\left(k_{\mu} k_{\nu} / m^{2}\right) \mathrm{e}^{i k \cdot x}, & k^{2}=-m^{2}, \\
h_{\mu \nu}^{(\alpha)}=\zeta_{\mu \nu}^{(\alpha)}(k) \mathrm{e}^{-i k \cdot x}, & k^{2}=0,
\end{array}
$$

where $\zeta_{\mu \nu}^{(\alpha)}(k)$ is the polarization tensor of a graviton with polarization $\alpha$ in volume gauge. The two gauge conditions (4.1) and (4.2) become for the field $h_{\mu \nu}$ :

$$
h_{\mu}^{\mu}=0, \quad \partial_{\mu} \partial^{\lambda} h_{\lambda \nu}-\partial_{\nu} \partial^{\lambda} h_{\lambda \mu}=0 .
$$

They are fulfilled by the polarization tensors of the two graviton states which satisfy (cf. ref. [22]):

$$
\zeta_{\mu}^{\mu}=0, \quad k^{\mu} \xi_{\mu \nu}=0 .
$$

Using the expressions (4.23b) and (4.23c) for the projection operators $P^{(2)}$ and $P^{(0)}$ one easily verifies the relations

$$
\begin{gathered}
h^{(\alpha) \mu \nu^{*}} P_{\mu \nu \lambda \tau}^{(2)} h^{(\beta) \lambda \tau}=\zeta_{\mu \nu}^{(\alpha)^{*}} \zeta^{(\beta) \mu \nu}, \\
h^{(\alpha) \mu \nu^{*}} P_{\mu \nu \lambda \tau}^{(2)} D^{\lambda \tau}=0 \\
h^{(\alpha) \mu \nu^{*}} P_{\mu \nu \lambda \tau}^{(0)} D^{\lambda \tau}=0 \\
D^{\mu \nu^{*}} P_{\mu \nu \lambda \tau}^{(0)} D^{\lambda \tau}=1 .
\end{gathered}
$$

Hence the propagator (4.23) describes indeed a massless graviton and a massive scalar particle, the dilaton. It remains to be shown, however, that there are no additional states in the physical sector of the Hilbert space. Because of the higher derivatives in the lagrangian (4.22) this is not obvious. We have performed such an analysis for a similar theory where conditions (4.2) are replaced by equations which contain an auxiliary field and only first-order derivatives. In this case we found that the physical Hilbert space indeed contains no additional states (cf. [17]).

We conclude that it is possible to describe graviton and dilaton together by means of the symmetric tensor $g_{\mu \nu}$ and an action with smaller gauge invariance. The corresponding lagrangian (3.17) is invariant only under coordinate transformations which preserve the volume. This description of graviton and dilaton is reminiscent of the construction of these states in string theories [18]. 


\section{Vacuum curvature}

Vacuum states of the theory are characterized by stationary points of the effective action for which scalar fields and curvature have constant values. The relevant part of the action (3.17) reads

$$
\begin{aligned}
S=\int \mathrm{d}^{4} x \sqrt{g}( & -\frac{\kappa}{2} R-\frac{f^{2}}{2} g^{\mu \nu} \frac{1}{\sqrt{g}} \partial_{\mu} \sqrt{g}^{1 / 4} \partial_{\nu} \sqrt{g}^{1 / 4} \\
& \left.-g^{\mu \nu} \partial_{\mu} \phi^{\dagger} \partial_{\nu} \phi-\bar{V}\left(\phi^{\dagger} \phi\right)+\frac{1}{4} \Delta\left(\phi^{\dagger} \phi\right) \ln \sqrt{g}\right),
\end{aligned}
$$

where the potential $\bar{V}\left(\phi^{\dagger} \phi\right)$ is given by eq. (2.14). Let us first consider the variation of the action under the infinitesimal coordinate transformation

$$
\begin{gathered}
g_{\mu \nu} \rightarrow g_{\mu \nu}+\delta g_{\mu \nu}, \\
\delta g_{\mu \nu}=\partial_{\mu} \varepsilon^{\lambda} g_{\lambda \nu}+\partial_{\nu} \varepsilon^{\lambda} g_{\mu \lambda}+\varepsilon^{\lambda} \partial_{\lambda} g_{\mu \nu},
\end{gathered}
$$

where $\varepsilon^{\mu}=x^{\mu}-x^{\prime \mu}$. Since only the second and the last term of the action (5.1) are not invariant under the transformation (5.2) one obtains with

$$
\delta \sqrt{g}=\varepsilon^{\mu} \partial_{\mu} \sqrt{g}+\sqrt{g} \partial_{\mu} \varepsilon^{\mu}
$$

after partial integration (cf. eq. (4.14))

$$
\delta S=-\frac{1}{4} \int \mathrm{d}^{4} x \partial_{\mu}\left(\sqrt{g}\left(\Delta+f^{2} \square \ln \sqrt{g}^{1 / 4}\right)\right) \varepsilon^{\mu} .
$$

For solutions of the equations of motion $\delta S$ vanishes for arbitrary $\varepsilon^{\mu}$ and we obtain from (5.4)

$$
\partial_{\mu}\left(\sqrt{g}\left(\Delta+f^{2} \square \ln \sqrt{g}^{1 / 4}\right)\right)=0 .
$$

This is precisely the condition (cf. (4.19)) which is required so that the BRS operator is nilpotent on-shell.

Variation of the action (5.1) with respect to $g_{\mu \nu}$ yields the equations of motion for the gravitational field:

$$
\begin{aligned}
R_{\mu \nu}-\frac{1}{2} g_{\mu \nu} R= & \left(f^{2 / \kappa}\right)\left(-\partial_{\mu} \ln \sqrt{g}^{1 / 4} \partial_{\nu} \ln \sqrt{g}^{1 / 4}\right. \\
& \left.+\frac{1}{2} g_{\mu \nu}\left(g^{\lambda \tau} \partial_{\lambda} \ln \sqrt{g}^{-1 / 4} \partial_{\tau} \ln \sqrt{g}^{1 / 4}-\frac{1}{2} \square \ln \sqrt{g}^{1 / 4}\right)\right) \\
& +(1 / \kappa)\left(T_{\mu \nu}-\frac{1}{4} g_{\mu \nu}(1+\ln \sqrt{g}) \Delta\left(\phi^{\dagger} \phi\right)\right),
\end{aligned}
$$


where $T_{\mu \nu}$ is the energy-momentum tensor of the scalar field:

$$
T_{\mu \nu}=-\left(\partial_{\mu} \phi^{\dagger} \partial_{\nu} \phi+\partial_{\nu} \phi^{\dagger} \partial_{\mu} \phi\right)+g_{\mu \nu}\left(g^{\lambda \tau} \partial_{\lambda} \phi^{\dagger} \partial_{\tau} \phi+\bar{V}\left(\phi^{\dagger} \phi\right)\right)
$$

From eq. (5.6) one obtains for the curvature scalar

$$
\begin{aligned}
R= & -\left(f^{2} / \kappa\right)\left(g^{\lambda \tau} \partial_{\lambda} \ln \sqrt{g}^{1 / 4} \partial_{\tau} \ln \sqrt{g}^{1 / 4}-\square \ln \sqrt{g}^{-1 / 4}\right) \\
& -(1 / \kappa)\left(T_{\mu}^{\mu}-(1+\ln \sqrt{g}) \Delta\left(\phi^{\dagger} \phi\right)\right) .
\end{aligned}
$$

The equation of motion for the scalar field $\phi$ reads:

$$
\square \phi-\frac{\partial}{\partial \phi^{\dagger}}\left(\bar{V}\left(\phi^{\dagger} \phi\right)-\frac{1}{4} \Delta\left(\phi^{\dagger} \phi\right) \ln \sqrt{g}\right)=0 .
$$

Vacuum states of the theory are given by solutions of eqs. (5.8) and (5.9) for which the scalar fields $\phi$ and $\sqrt{g}$, and the curvature scalar $R$ take constant values. The space-time with constant curvature is de Sitter space whose metric has the canonical form ${ }^{\star}$ :

$$
g_{\mu \nu}(x)=\eta_{\mu \nu}+\frac{\varepsilon}{1-\varepsilon x^{2}} \eta_{\mu \lambda} x^{\lambda} \eta_{\nu \tau} x^{\tau}
$$

with

$$
\begin{aligned}
x^{2} & =\eta_{\mu \nu} x^{\mu} x^{\nu}, \\
R_{\mu \nu \rho \sigma} & =\varepsilon\left(g_{\mu \sigma} g_{\nu \rho}-g_{\mu \rho} g_{\nu \sigma}\right), \\
R & =-12 \varepsilon .
\end{aligned}
$$

One easily obtains from eq. (5.10)

$$
g=-\operatorname{det}\left(g_{\mu \nu}\right)=\frac{1}{1-\varepsilon x^{2}} .
$$

It is possible to perform a coordinate transformation, which leaves the Ricci scalar $R$ invariant, such that the new metric tensor $g_{\mu \nu}^{\prime}$ has constant determinant:

$$
\operatorname{det}\left(g_{\mu \nu}^{\prime}\right)=\operatorname{det}\left(g_{\mu \nu}\right)\left(\operatorname{det}\left(\partial x / \partial x^{\prime}\right)\right)^{2} \stackrel{!}{=} \mathrm{e}^{4 \alpha}, \quad \alpha=\text { const }
$$

\footnotetext{
$\star$ See, for instance, ref. [22].
} 
A non-singular transformation satisfying this condition is easily constructed. After some algebra we obtain:

$$
\begin{gathered}
x^{\mu}=\sqrt{\lambda\left(x^{\prime 2}\right)} x^{\prime \mu}, \\
\left(2+\varepsilon \lambda\left(x^{\prime 2}\right) x^{\prime 2}\right) \sqrt{1-\varepsilon \lambda\left(x^{\prime 2}\right) x^{\prime 2}}=2-\frac{3}{4} \varepsilon^{2} e^{4 \alpha}\left(x^{\prime 2}\right)^{2} .
\end{gathered}
$$

For small $x^{\prime 2}$ one has

$$
\lambda\left(x^{\prime 2}\right)=1+\mathrm{O}\left(\mathrm{e}^{2 \alpha} \varepsilon x^{\prime 2}\right) .
$$

The final expression for the transformed metric tensor reads:

$$
g_{\mu \nu}^{\prime}\left(x^{\prime}\right)=\lambda\left(x^{\prime 2}\right) \eta_{\mu \nu}+\frac{\mathrm{e}^{8 \alpha}-\lambda^{4}\left(x^{\prime 2}\right)}{\lambda^{3}\left(x^{\prime 2}\right)} \frac{x_{\mu}^{\prime} x_{\nu}^{\prime}}{x^{\prime 2}}
$$

This metric, whose determinant is constant by construction, describes a space-time of constant curvature $R^{\prime}=-12 \varepsilon$.

For constant scalar fields $\phi_{0}$ one has

$$
T^{\mu}{ }_{\mu}=4 \bar{V}\left(\phi_{0}^{\dagger} \phi_{0}\right) \text {. }
$$

Inserting (5.16) into eqs. (5.8) and (5.9) one obtains the extremum conditions

$$
\begin{aligned}
\bar{V}\left(\phi_{0}^{\dagger} \phi_{0}\right)-3 \kappa \varepsilon-\Delta\left(\phi_{0}^{\dagger} \phi_{0}\right)\left(\alpha+\frac{1}{4}\right) & =0, \\
\left.\frac{\partial}{\partial \phi^{\dagger}}\left(\bar{V}\left(\phi^{\dagger} \phi\right)-\Delta\left(\phi^{\dagger} \phi\right) \alpha\right)\right|_{\phi=\phi_{0}} & =0 .
\end{aligned}
$$

These equations are almost identical to the corresponding equations (2.16) in flat space, with $\alpha=\sigma_{0} / f$. The only difference is the appearance of the additional constant $3 \kappa \varepsilon$, i.e., eq. (5.18) corresponds to eq. (2.16b) in flat space with a modified constant in the effective potential (cf. eq. (2.1d)):

$$
a_{\varepsilon}^{4}=a^{4}-3 \kappa \varepsilon .
$$

Within the model discussed, the only constraint on the constant $a_{\varepsilon}$ is eq. (2.17) which stems from the requirement of the existence of a solution of eqs. (2.16) with finite values of $\phi_{0}$ and $\sigma_{0}$. If one does not allow for an accidental cancellation between the two terms $a$ and $3 \kappa \varepsilon$, one obtains for $\varepsilon$ the constraint

$$
|\varepsilon|<\mu^{4} / \kappa,
$$

which corresponds to the naive expectation. 
So far we have studied the ground state curvature in the model with restricted coordinate invariance. As we have discussed in sect. 3, an alternative model for "dilatons" in curved space is the Brans-Dicke theory. From eqs. (3.1b), (3.7) and (3.21) one obtains the equations of motion

$$
\begin{gathered}
R=-(1 / \kappa)\left(g^{\mu \nu} \partial_{\mu} \sigma \partial_{r} \sigma+\bar{T}^{\mu}{ }_{\mu}\right), \\
f \square \sigma=\bar{T}_{\mu}^{\mu}-\mathrm{e}^{4 \sigma / f} \Delta\left(\phi^{\dagger} \phi\right), \\
\square \phi+\frac{2}{f} \partial^{\mu} \sigma \partial_{\mu} \phi-\mathrm{e}^{2 \sigma / f} \frac{\partial}{\partial \phi^{\dagger}}\left(\bar{V}\left(\phi^{\dagger} \phi\right)-\Delta\left(\phi^{\dagger} \phi\right) \sigma / f\right)=0,
\end{gathered}
$$

where the energy-momentum tensor depends now on $\phi$ and $\sigma$ :

$$
\begin{aligned}
\bar{T}_{\mu \nu}= & -\mathrm{e}^{2 \sigma / f}\left(\partial_{\mu} \phi^{\dagger} \partial_{\nu} \phi+\partial_{\nu} \phi^{\dagger} \partial_{\mu} \phi-g_{\mu \nu} g^{\lambda \tau} \partial_{\lambda} \phi^{\dagger} \partial_{\tau} \phi\right) \\
& +g_{\mu \nu} \mathrm{e}^{4 \sigma / f}\left(\bar{V}\left(\phi^{\dagger} \phi\right)-\Delta\left(\phi^{\dagger} \phi\right) \sigma / f\right) .
\end{aligned}
$$

For constant fields $\left(\phi_{0}, \boldsymbol{\sigma}_{0}\right)$ eqs. (5.23) and (5.24) are identical to the flat space equations (2.16). Eqs. (5.22) and (5.23) imply

$$
R_{0}=-(1 / \kappa) \mathrm{e}^{4 \sigma_{0} / f} \Delta\left(\phi_{0}^{\dagger} \phi_{0}\right)
$$

i.e., the ground state curvature is uniquely determined, and its size is given by the vacuum expectation value of the anomaly. This unwanted result can be avoided if one eliminates the anomaly term in the effective potential by adding the Wess-Zumino term (2.22). Then, eqs. (5.22) and (5.23) yield $R_{0}=0$. However, the existence of a constant solution $\sigma_{0}$ requires fine-tuning of the constant in the effective potential as discussed in sect. 2 .

Eq. (5.26) has recently been derived by Coughlan et al. [10] in a general analysis on the role of dilatation symmetry for the cosmological constant problem. We agree with the main conclusion of the paper that spontaneously broken scale invariance does not automatically, i.e., without any fine-tuning of parameters, lead to vanishing ground state curvature. Nevertheless, the presence of dilatons in a theory has some interesting implications for the cosmological constant problem: In a Brans-Dicke theory, vanishing curvature is equivalent to the existence of a constant solution $\left(\phi_{0}, \sigma_{0}\right)$ of the equations of motion, which is usually required as ground state. In theories with restricted coordinate invariance, where the dilaton is identified with the conformal factor of the metric tensor, for a certain range of parameters in the potential there always exists a constant solution $\left(\phi_{0}, \sigma_{0}\right)$ with vanishing curvature, which, however, is not the unique ground state. Furthermore, the scalar potential, 
which occurs in both models of dilatons in curved space, has turned out to be interesting with respect to the cosmological time dependence of the vacuum energy density of the scalar fields, as recently discussed by Wetterich [23], and Ratra and Peebles [24]. We will discuss this issue, as well as phenomenological constraints on light scalar particles [25] in more detail elsewhere.

\section{Summary}

The starting point of this paper was the scale invariant standard model where dilatations are realized nonlinearly by means of a Goldstone field, the dilaton. Quantum corrections break the classical scale invariance, and a unique ground state exists without fine-tuning of parameters in the effective potential. The dilaton acquires a small mass which, like the vacuum energy density, is determined by the vacuum expectation value of the anomaly.

The extension of this model to curved space-time is not unique. The main result of this paper is a description of the dilaton as part of the metric tensor which also contains the graviton. The corresponding action is invariant only under restricted coordinate transformations which preserve the volume. This is reminiscent of the construction of dilaton and graviton states in string theories. The dilaton is identified with the conformal factor of the metric and transforms inhomogeneously under dilatations which remain an anomalous global symmetry also in curved space. This theory is known to be classically almost equivalent to Einstein's theory of gravity with an additional scalar field. The only difference concerns the curvature of the ground state which is no longer determined by a parameter of the lagrangian. It rather appears as integration constant which is related to the vacuum expectation value of the dilaton field. We have also constructed BRS invariant gauge fixing and ghost lagrangians for the restricted gauge invariance and explicitly evaluated the propagator of the dilaton-graviton system.

Although our treatment of dilatons in curved space appears to be theoretically consistent, it is not clear that it is relevant as part of an effective low energy lagrangian of a fundamental theory. An alternative is a theory with general coordinate invariance and anomalous global Weyl invariance. Here the "dilaton" becomes the Goldstone boson of the global Weyl symmetry. However, this theory is only consistent if the vacuum expectation value of the conformal anomaly vanishes, which is not the case in the standard model. Another interesting alternative is the Brans-Dicke theory where only part of the action is invariant under Weyl transformations. Even in the absence of the conformal anomaly graviton and "dilaton" kinetic terms break Weyl invariance. In the Brans-Dicke theory the vacuum expectation value of the anomaly leads to a nonvanishing ground state curvature.

The dilaton field plays an important role for the vacuum energy density and therefore also for the curvature of the ground state. The direct relation between dilaton vacuum expectation value and ground state curvature in models with 
restricted coordinate invariance appears particularly interesting. This will be discussed in more detail elsewhere.

We are indebted to M. Kreuzer, R.D. Peccei and C. Wetterich for helpful discussions throughout the course of this work.

\section{Appendix}

In ref. [9] we have obtained expression (3.8) for the effective potential in a gravitational background field (cf. eqs. $(2.14,2.15))$ :

$$
\sqrt{g} V\left(\phi^{\dagger} \phi, \sigma, \sqrt{g}\right)=\sqrt{g} \mathrm{e}^{4 \sigma / f}\left(\bar{V}\left(\phi^{\dagger} \phi\right)-\Delta\left(\phi^{\dagger} \phi\right)\left(\sigma / f+\frac{1}{4} \ln \sqrt{g}\right)\right) .
$$

On the other hand gravitational background field calculations using dimensional regularization yield in the case $\sigma=0[26,27]$ :

$$
\sqrt{g} \tilde{V}\left(\phi^{\dagger} \phi, \sqrt{g}\right)=\sqrt{g} \bar{V}\left(\phi^{\dagger} \phi\right)
$$

i.e., the term $\Delta\left(\phi^{\dagger} \phi\right) \ln \sqrt{g}$ does not appear.

The difference between (3.8) and (A.1) can be easily understood as follows. Let us consider for simplicity a single scalar field $\phi$. The starting point of the calculation in dimensional regularization is:

$$
S_{0}[\phi, g]=-\int \mathrm{d}^{n} x \sqrt{g}\left(\frac{1}{2} g^{\mu \nu} \partial_{\mu} \phi \partial_{\nu} \phi+V_{0}(\phi)\right)
$$

In the special case of a conformally flat metric,

$$
g_{\mu \nu}=\mathrm{e}^{2 \alpha} \eta_{\mu \nu},
$$

the action (A.2) becomes:

$$
S_{0}[\phi, g]=-\int \mathrm{d}^{n} \chi \mathrm{e}^{n \alpha}\left(\frac{1}{2} \mathrm{e}^{-2 \alpha} \eta^{\mu \nu} \partial_{\mu} \phi \partial_{\nu^{\prime}} \phi+V_{0}(\phi)\right) .
$$

For constant fields $\phi$ the one-loop correction is given by ref. [26]:

$$
\begin{aligned}
S_{1}[\phi, g]= & -\frac{1}{(4 \pi)^{2}} \int \mathrm{d}^{n} x \mathrm{e}^{n \alpha}\left[\frac{1}{n-4}+\frac{1}{2}\left(\gamma_{\mathrm{E}}-\ln 4 \pi+\ln \frac{m^{2}(\phi)}{M^{2}}\right)\right] \\
& \times \frac{4}{n(n-2)}\left(m^{2}(\phi)\right)^{2},
\end{aligned}
$$


where

$$
m^{2}(\phi)=\partial^{2} V_{0}(\phi) / \partial \phi^{2}
$$

and $\gamma_{E}$ is Euler's constant. After renormalization one obtains the ColemanWeinberg effective potential:

$$
\begin{aligned}
S_{1}^{(\mathrm{R})}[\phi, g] & =-\frac{1}{(8 \pi)^{2}} \int \mathrm{d}^{4} x \sqrt{g}\left(m^{2}(\phi)\right)^{2} \ln \frac{m^{2}(\phi)}{M^{2}} \\
& \equiv-\int \mathrm{d}^{4} x \sqrt{g} V_{1}(\phi) .
\end{aligned}
$$

In the case of an additional dilaton field the classical action reads in four dimensions (cf. (3.1b)):

$$
\bar{S}_{0}[\phi, \sigma, g]=-\int \mathrm{d}^{4} x \sqrt{g} \mathrm{e}^{4 \sigma / f}\left(\frac{1}{2} g^{\mu \nu} \mathrm{e}^{-2 \sigma / f} \partial_{\mu} \phi \partial_{\nu} \phi+V_{0}(\phi)\right)
$$

There are four different ways in which this action can be continued to $n$ dimensions. They correspond to broken or unbroken Weyl and general coordinate invariance. In the case of a conformally flat metric the result of ref. [9] corresponds to the choice

$$
\bar{S}_{0}[\phi, \sigma, g]=-\int \mathrm{d}^{n} x \mathrm{e}^{4(\alpha+\sigma / f)}\left(\frac{1}{2} \eta^{\mu v} \mathrm{e}^{-2(\alpha+\sigma / f)} \partial_{\mu} \phi \partial_{\nu} \phi+V_{0}(\phi)\right)
$$

i.e., the conformal factor in the metric and the dilaton field are treated symmetrically. From eqs. (A.4) and (A.8) one obtains ${ }^{\star}$ :

$$
\bar{S}_{1}^{(\mathrm{R})}[\phi, \sigma, g]=-\int \mathrm{d}^{4} x \sqrt{g} \mathrm{e}^{4 \sigma / f}\left(V_{1}(\phi)-\Delta_{1}(\phi)\left(\sigma / f+\frac{1}{4} \ln \sqrt{g}\right)\right)
$$

where

$$
\Delta_{1}(\phi)=M \partial V_{1}(\phi) / \partial M
$$

is the conformal anomaly. In eq. (A.9) general coordinate invariance is broken by the anomaly.

An alternative continuation to $n$ dimensions, which maintains general coordinate invariance, reads for conformally flat metric:

$$
\tilde{S_{0}}[\phi, \sigma, g]=-\int \mathrm{d}^{n} x \mathrm{e}^{n \alpha+4 \sigma / f}\left(\frac{1}{2} \eta^{\mu \nu} \mathrm{e}^{-2(\alpha+\sigma / f)} \partial_{\mu} \phi \partial_{\nu} \phi+V_{0}(\phi)\right)
$$

\footnotetext{
* The same result is obtained in a direct evaluation of the one-loop correction to eq. (A.8) [28]
} 
The corresponding one-loop correction is given by

$$
\tilde{S_{1}^{(R)}}[\phi, \sigma, g]=-\int \mathrm{d}^{4} x \sqrt{g} \mathrm{e}^{4 \sigma / f}\left(V_{1}(\phi)-\Delta_{1}(\phi) \sigma / f\right)
$$

As discussed in sect. 3 this asymmetric treatment of the conformal factor in the metric and the dilaton field is only consistent if the vacuum expectation value of the conformal anomaly vanishes.

Of course, it is also possible to maintain Weyl and general coordinate invariance. All four options differ only by local Wess-Zumino terms, and it is a physical question which symmetries one requires the renormalized effective action to have.

\section{References}

[1] G. Mack, in Nonperturbative quantum field theory, Cargese lectures 1987, ed. G. 't Hooft et al. (Plenum Press, New York) to appear

[2] M.B. Green, J.H. Schwarz and E. Witten, Superstring theory (Cambridge Univ. Press, Cambridge. 1987)

[3] S. Coleman, Dilatations, in Aspects of Symmetry, (Cambridge Univ. Press, Cambridge. 1985) p. 67

[4] B. Zumino, Effective lagrangians and broken symmetries, in Lectures on elementary particles and quantum field theory, eds. S. Deser, M. Grisaru, H. Pendleton (Brandeis University, 1970) vol. II

[5] H.P. Nilles, Phys. Rep. 110 (1984) 1:

N. Dragon, U. Ellwanger and M.G. Schmidt, Prog. in Part. and Nucl. Physics 18 (1987) 1

[6] C. Brans and R.H. Dicke, Phys. Rev. 124 (1961) 925

[7] R.D. Peccei and H.R. Quinn, Phys. Rev. Lett. 38 (1977) 1440; Phys. Rev. D16 (1977) 1791

[8] R.D. Peccei, J. Solà and C. Wetterich, Phys. Lett. B195 (1987) 183

[9] W. Buchmüller and N. Dragon, Phys. Lett. B195 (1987) 417

[10] G.D. Coughlan, I. Kani, G.G. Ross and G. Segrè, preprint CERN-TH-5014 (1988)

[11] F. del Aguila and G.D. Coughlan, Phys. Lett. B180 (1986) 25

[12] S. Coleman and E. Weinberg, Phys. Rev. D7 (1973) 1888

[13] R. Crewther, Phys. Rev. Lett. 28 (1972) 1421;

M.S. Chanowitz and J. Ellis, Phys. Lett. 40B (1972) 397:

J.C. Collins, Phys. Rev. D14 (1976) 1965

[14] S. Weinberg, Phys. Rev. Lett. 36 (1976) 294:

A.D. Linde, JETP Lett. 23 (1976) 64

[15] J. Wess and B. Zumino, Phys. Lett. B37 (1971) 95

[16] L. Bonora, P. Pasti and M. Tonin, Phys. Lett. B149 (1984) 346

[17] W. Buchmüller and N. Dragon, Gauge fixing and the cosmological constant, Phys. Lett. B, to appear

[18] J. Scherk and J.H. Schwarz, Nucl. Phys. B81 (1974) 118

[19] J.J. van der Bij, H. van Dam and J.Y. Ng. Physica 116A (1982) 307

[20] W. Buchmüller and N. Dragon, Phys. Lett. B207 (1988) 292

[21] N. Dragon and M. Kreuzer, Z. Phys. C41 (1988) 485

[22] S. Weinberg, Gravitation and cosmology (John Wilcy and Sons, New York, 1972)

[23] C. Wetterich, Nucl. Phys. B302 (1988) 668

[24] B. Ratra and P.J.E. Peebles, Phys. Rev. D37 (1988) 3406

[25] J. Ellis, N.C. Tsamis and M. Voloshin, Phys. Lett. B194 (1987) 291

[26] J. Guven, Phys. Rev. D35 (1987) 2378

[27] L.S. Brown, private communication

[28] D. Boedecker, Diplom thesis (Hannover, 1989) 